\title{
3 Staatliche Grenzen im Kontext räumlicher Strukturierungen des Sozialen
}

Unter den zahlreichen Mitteln, mit denen Gesellschaften sich organisieren, soziale Ordnung erzeugen und sichern, sind territorial-räumliche Grenzen eines der machtvollsten, symbolträchtigsten und umstrittensten. ${ }^{1}$ Die nationalstaatliche Grenze ist eng verbunden mit einem Territorialisierungsprinzip, das im 19. Jahrhundert zunächst im westlichen Europa hegemonial wird: das Prinzip fixierter, distinkter, wechselseitig exklusiver und (schließlich) demarkierter Räume staatlicher Souveränität. Staatsgrenzen wurden - bei aller Vielfalt ihrer konkreten Erscheinungsformen - zum Mittel wie auch zum Ausdruck einer als spezifisch neuzeitlich verstandenen Raumordnung: der Ordnung der „umzäunten Machtcontainer“ souveräner Staaten. „An kaum einer Stelle“, wie Reuber ${ }^{3}$ betont, „treten die Machtwirkungen gesellschaftlicher Räumlichkeit so eindrucksvoll hervor wie hier.“

Die nordamerikanische frontier ist ein instruktives Beispiel für die gewaltsame Durchsetzung von Territorialisierungen im Kontext sich formierender Staatlichkeit. Die frontier war weniger eine Grenze als vielmehr eine Zone unklarer bzw. wechselnder Machtverhältnisse, die nicht primär durch eine lineare Abgrenzung territoriale Gebilde definiert ist als vielmehr - in wechselnden Gemengelagen und Interessenkoalitionen „staatlicher“ und „nicht-staatlicher“ Akteur`innen durch den permanenten Kampf um die Aneignung von Land und die anschließende Sicherung der Kontrolle über dasselbe. ${ }^{4}$ Die von Reuber angesprochene Räumlichkeit entfaltet sich nach innen in all den vielfältigen Formen, in denen Staatlichkeit das Alltagsleben durchdringt und gestaltet oder zumindest Strukturierungsansprüche erhebt ${ }^{5}$ - und dabei auch mit anderen Verräumlichungen interferiert. Nach außen entfaltet sich diese Raumordnung im Hinblick auf die je eigene, mit dem Territorium gewissermaßen amalgamierte Souveränität, die in der linearen, erdräumlich fixierten territorialen Grenzen kulminiert.

1 Siehe hierzu als historischen Querschnitt W. von Bredow, Grenzen. Eine Geschichte des Zusammenlebens vom Limes bis Schengen, Berlin: Theiss, 2014.

2 Im Original „bounded power-container“, A. Giddens, Social Theory and Modern Sociology, hier S. 171 .

3 S. Reuber, Politische Geographie, Paderborn: Schöningh (UTB), 2012.

4 Siehe als Überblick M. Anderson, Frontiers: Territory and State Formation in the Modern World, Hoboken: John Wiley \& Sons, 2013.

5 Vgl. J. Painter, „Prosaic Geographies of Stateness“. 
Räumliche Ordnungen des Sozialen werden aber nicht nur durch das Agieren von Staatsapparaten und Kriegsmaschinerien geprägt, sondern auch durch ökonomische Verflechtungen bestimmt, ${ }^{6}$ für deren zirkulatorische Logiken - die Mobilitäten von Waren, Kapital und Arbeitskraft - Staatsgrenzen auf vielfältige Weise relevant werden können. Ökonomische Interessen (z. B. das Interesse an einem ungehinderten Kapital- und Warenverkehr) und politische Interessen (z. B. Wahrung der souveränen Rechte des einzelnen Staates, Interesse an der Kontrolle der Bewegung von Menschen, Interesse an Steuer- und Zolleinnahmen) können dabei in ein Spannungsverhältnis geraten. Die weitgehende Aufhebung innereuropäischer Grenzen durch die Schengener Abkommen, die eine zentrale Voraussetzung für die Schaffung eines Binnenmarkts war, stellt einen besonders prominenten, derzeit massiv kritisierten Versuch dar, den Widerspruch zwischen politischer und ökonomischer Verräumlichung zu lösen.

Staatsgrenzen sind im Hinblick auf ihre Effekte ausgesprochen ambivalent: Grenzen markieren und sichern staatliche Territorien und gehören damit zum Repertoire räumlich-institutioneller Praktiken, die gesellschaftliche Ordnung als (national-)staatliche Ordnung herstellen. Strategische Ansprüche, nationalstaatliche Verhältnisse zu überwinden (wie z. B. durch die Etablierung des europäischen Binnenmarkts mittels der Einheitlichen Europäischen Akte) wie auch umgekehrt diese gegen Nachbarstaaten oder aber in bestimmte Richtungen nach außen abzuschotten (z. B. Abgrenzung der DDR gegen die BRD, aber auch phasenweise - gegenüber Polen; Abgrenzung Nordkoreas gegen China, Russland und v. a. Japan), basieren essentiell auf der Formierung und praktischen Durchsetzung eines entsprechenden Grenzregimes. Die Schlüsselfunktion von Grenzregimes in Bezug auf die Organisation gesellschaftlicher Ordnungen wird nicht zuletzt in den Verhandlungen zum Austritt des Vereinigten Königreichs aus der EU deutlich, in denen die Frage einer zukünftigen Gestaltung der (Land-)Grenze zwischen der Republik Irland und dem Vereinigten Königreich zu einem der schwierigsten Kapitel gehört. ${ }^{7}$ Regionalisierungsprozesse wirken sich mithin nicht nur auf die konkrete Gestaltung von Grenzregimes aus, indem etwa Zollbestimmungen vereinheitlicht und Freihandelszonen eingerichtet werden. Sie wirken sich auch auf die Möglichkeit der Definition der Bedingungen, d. h. - im Falle der Schengenraums - der weitgehende Verzicht auf bilaterale Aushandlungen zugunsten eines homogenisierten Kanons von Regeln und Praktiken der Durchsetzung derselben.

6 K. Ohmae, The Borderless World. Power and Strategy in the Interlinked Economy, New York: HarperBusmess, 1990.

7 Zum Zeitpunkt der Fertigstellung des Manuskripts (September 2018) hatte die britische Regierung immer noch kein schlüssiges Konzept präsentiert. 
In seiner 2012 erschienenen Politischen Geographie versteht Paul Reuber ${ }^{8}$ Grenzen als Mittel sozialer Strukturierung, das zwar „eine räumliche, eine physisch-materielle Komponente enthalten“9 könne, die aber nicht als Ursache oder determinierendes Moment verstanden werden dürfe. Diese Argumentationslinie lässt sich zu einem Diktum des Soziologen Georg Simmel zurückverfolgen, das besagt, dass eine Grenze „nicht eine räumliche Tatsache mit soziologischen Wirkungen [ist], sondern eine soziologische Tatsache, die sich räumlich formt“ ". ${ }^{10}$ Beide Zitate, zwischen denen mehr als ein Jahrhundert liegt, verweisen auf ein fundamentales Problem im Verständnis politischer Grenzen: Auch wenn sie sich visuell/medial und im individuellen Erleben eines Grenzübertritts gerade durch ihre physische Gestalt aufdrängen, sind Grenzen primär ein Produkt gesellschaftlicher Verhältnisse - der gewaltsamen Abgrenzung von Machtsphären wie auch der Verdichtung sozialer Beziehungen und der Integration zirkulatorischer Prozesse - die sich physisch-materieller, „landschaftlicher“ Markierungen bedienen können. Eine Grenze, die z. B. einem Flusslauf, einem Hochgebirgsgrat, einer Wasserscheide oder einer Küste folgt, anhand bestimmter Qualitäten der naturräumlichen Ausstattung verstehen und erklären $\mathrm{zu}$ wollen, ist daher wenig sinnvoll. Ein Hochgebirgsgrat determiniert nicht die Grenze zwischen zwei Staaten, er kann sie bestenfalls markieren. Gleichwohl ist es natürlich berechtigt der Frage nachzugehen, wie und unter welchen Bedingungen ein Grenzverlauf in einer bestimmten Weise (friedlich oder gewaltsam) festgesetzt worden ist, warum ein Grenzverlauf sich an bestimmten landschaftlichen Gegebenheiten orientiert ${ }^{11}$ und warum es in einem bestimmten historischen Moment überhaupt notwendig wurde, einer politischen Entität eine eindeutige, d. h. linear begrenzte territoriale Gestalt zu geben. ${ }^{12}$ Ebenso wie eine Grenze sich an naturräumlichen Gegebenheiten orientieren kann, kann sie aber auch quer zu diesen verlaufen. ${ }^{13}$

8 Reuber, Politische Geographie, S. 61.

9 Ebd.

10 G. Simmel, „Der Raum und die räumliche Ordnung der Gesellschaft“, in: M. Eigmüller, G. Vobruba (Hrsg.), Grenzsoziologie. Wiesbaden: VS, 2006, S. 15-23 (Originaltext 1908 erschienen). 11 Die Grenze zwischen der nach Unabhängigkeit strebenden Republik Abchasien und Georgien verläuft durch den Enguri/Egry; er markiert die Waffenstillstandslinie, auf die sich die Kriegsparteien 1994 einigen konnten. Dennoch ist der Fluss keine „natürliche“ Grenze, sondern ein an einem bestimmten landschaftlichen Element orientiertes Ergebnis gewalttätiger Aushandlungsprozesse.

12 Ausgehend von einer etymologischen Untersuchung zum Zusammenhang von terrain, terror und territory beschäftigen sich vor allem die Arbeiten von Stuart Elden (S. Elden, „Land, Terrain, Territory“, Progress in Human Geography 34 [2010] 6, S. 799-817; S. Elden, „Governmentality, Calculation, Territory“, Environment and Planning D: Society and Space 25 [2007] 3, S. 562-580) 
Insbesondere im Kontext politisch-geographischer Praktiken und Wissensproduktionen gelten Territorien und Grenzen - im Sinne des englischen boundary - vielfach als komplementäre, in die Landschaft eingeschriebene Konstruktion: die lineare Form der Grenze umschließt das zweidimensionale Territorium. ${ }^{14}$ Von einer „natürlichen“ Grenze zu sprechen stellt jedoch eine ontologische Verschiebung dar, weil es eine soziale, historische und damit auch kontingente Erscheinung zu einer natürlichen und damit in gewisser Weise unvermeidlichen und dem gesellschaftlichen Zugriff entzogenen, d. h. naturalisierten und reifizierten Tatsache macht. Diese Kritik stellt allerdings nicht die Wirkmächtigkeit der Verschiebung in Frage, sondern unterstreicht eher ihre Zähigkeit, die letztlich auch konzeptionellen Veränderungen häufig entgegensteht: Denn auch wenn die Annahme „natürlicher Grenzen“ wissenschaftlich problematisch und in Bezug auf ihren Erklärungsgehalt äußerst unbefriedigend ist, bedeutet das nicht, dass die Vorstellung einer Natürlichkeit von Grenzen irrelevant wäre - im Gegenteil:

mit dem Zusammenhang von militärischer Eroberung und der Definition eines Territoriums mittels linearer Grenzziehung.

13 Durch das Ferghana-Tal beispielsweise, das im Hinblick auf seine klimatischen, orographischen und hydrographischen Bedingungen wie auch im Hinblick auf die infrastrukturelle Erschließung und landwirtschaftliche Nutzung als Einheit wahrgenommen werden könnte, laufen die Staatsgrenzen von Usbekistan, Tadschikistan und Kirgistan. Zudem ist es bis in die angrenzenden Bergregionen hinein durchsetzt mit Exklaven: der tadschikischen Exklaven Sarwan in Usbekistan und Woruch in Kirgistan, den usbeskischen Exklaven So’x, Chong-Kara, Jangail und Shohimardon in Kirgisistan und der kirgisischen Exklave Barok in Usbekistan. Einige Exklaven, wie die zu Tadschikistan gehörende Siedlung West-Qal'acha/Kayragach in Kirgistan umfassen nicht vielmehr als eine Straßeneinmündung, einige Häuser und etwas angrenzendes Bergland (N. Megoran, The Border of Eternal Friendship? The Politics and Pain of Nationalism and Identity Along the Uzbekistan-Kyrgyzstan Ferghana Valley Boundary, 1999-2000, University of Cambridge, 2002, https://www.staff.ncl.ac.uk/nick.megoran/pdf/ nick_megoran_phd.pdf (abgerufen am 12. März 2019); R. Gabdulhakov, Geographical Exclaves of Fergana Valley: Do Good Fences Make Good neighbors? , o.J. [ca. 2014], http://osce-academy.net/upload/Policy_briefs/Policy_Brief_14.pdf (zuletzt eingesehen am 12. März 2019). Diese Exklaven sind keine Hinterlassenschaft der Auflösung der Sowjetunion, sondern bestehen wesentlich länger. Einige waren 1955 Gegenstand einer inter-republikanischer Kommission zur Demarkation von Grenzen, die ihre Arbeit aber nie beenden konnte (siehe Megoran, The Border of Eternal Friendship?, S. 39). Unter sowjetischer Herrschaft waren die Grenzen zwischen den SSR sowie rund um die Exklaven im Ferghana-Tal im Alltagsleben kaum spürbar; mit dem Zerfall der UdSSR änderte sich ihr Status und ihre alltägliche Relevanz jedoch beträchtlich. Einige Exklaven wurden mit Stacheldrahtzäunen und Straßenblockaden vom Umland abgetrennt; vgl. Megoran, The Border of Eternal Friendship?, S. $180 \mathrm{ff}$.

14 S. Elden, „Terror and Territory“, Antipode 39 (2007) 5, S. 821-845; S. Reuber, „Territorien und Grenzen“, in: J. Lossau, T. Freytag, R. Lippuner (Hrsg.), Schlüsselbegriffe der Kultur- und Sozialgeographie, Stuttgart: UTB, 2014, S. 192-197. 
Der physisch-materielle Raum bietet eine Vielzahl von Möglichkeiten, sozialweltliche Differenzierungen zu festigen und zugleich jenseits der sozialen Sphäre zu begründen. Er kann ausgedeutet werden, wenn es darum geht Raumordnungen herzustellen und zu plausibilisieren. Er kann - beispielsweise als Hochgebirgskamm - die Widerständigkeiten bieten, die es erleichtern, soziale Sphären voneinander $\mathrm{zu}$ trennen (und getrennt $\mathrm{zu}$ halten), um so - bei Bedarf ${ }^{15}$ - eindeutige Wirtschafts-, Einfluss- und Kontrollsphären zu definieren. Mit anderen Worten: Die in der Semantik der „natürlichen Grenze“ adressierte Aneignung des physisch-materiellen Raumes fungiert als eine Reifizierungs- und Naturalisierungsmaschinerie, mittels derer sozialweltliche Grenzziehungen nicht nur festgeschrieben werden, sondern zugleich von ihrem sozialweltlichen Ursprung abgetrennt und gereinigt werden können.

Die physische Markierung ist eine (arbiträre) Manifestation einer sozialen Grenzziehung, d. h. der physische, landschaftliche Raum bringt eine soziale Grenzziehung nicht hervor, kann sie aber - auf den ersten Blick oft hochplausibel - abbilden, sichtbar machen, damit aber auch reifizieren und festschreiben. Er kann dies aber nur aufgrund sozialer Akte, die ihm diese Funktion und Bedeutung zuschreiben. Die Arbitrarität solcher Aneignungsakte wird dann sichtbar, wenn sie sich ändern oder wenn es einander widersprechende Logiken der Aneignung gibt. Ein Fluss kann im Sinne der Separierung als „sinnvolle“ Grenzlinie erscheinen, weil er als eine sichtbare Unterbrechung der Kontinuität einer Landschaft gedeutet werden kann, deren Überwindung mit technischem, organisatorischem, finanziellem und zeitlichem Aufwand verbunden ist ${ }^{16}$. Er lässt sich wahrscheinlich auch besser kontrollieren allerdings als z. B. eine Feldkante. In der Logik des Wassermanagements, das sich zunehmend ${ }^{17}$ auf unterirdische Aquifere richtet, stellt eine am Oberflächenphänomen Flusslauf orientierte Grenze gerade eben keine „natürliche“ Grenze dar, sondern eine organisatorisch-

15 Eine eindeutige Fixierung ist keine notwendige Voraussetzung für Staatlichkeit, wohl aber ein Mittel, um Steuereinnahmen und Kontrolle über Bodenschätze u. ä. zu sichern; vgl. hierzu die Festlegung der Grenze zwischen Dänemark-Norwegen und Schweden-Finnland im 18. Jahrhundert, in S. Koch, „Sami-State Relations and its Impact on Reindeer Herding Across the NorwegianSwedish Border“, J. Miggelbrink u. a. (Hrsg.), Nomadic and Indigenous Spaces. Productions and Cognitions, Farnham: Ashgate, 2014.

16 Mit dann gelegentlich bizarren Effekten wie sie beispielsweise Ileana Stănculescu in ihrem Dokumentarfilm „Podul peste Tisa/The Bridge“ (2004) zeigt, vgl. J. Miggelbrink, „Crossing Lines, Crossed by Lines: Everyday Practices and Local Border Traffic in Schengen Regulated Borderlands“, in: R. Jones, C. Johnson (Hrsg.), Placing the Border in Everyday Life, Border Regions Series, Farnham: Ashgate, 2014.

17 Nicht zuletzt vor dem Hintergrund eines wachsenden Wasserbedarfs vieler Gesellschaften und des Klimawandels, der dazu führt, dass die Wasserversorgung an vielen Orten unzuverlässig wird. 
administrativ-politische Herausforderung, ${ }^{18}$ an der sich potenziell neue Konflikte entzünden können. ${ }^{19}$

Daraus resultiert, zweitens, dass nicht die „soziale Tatsache“ der Grenze im Zentrum des analytischen Interesses steht, sondern die sozialen Akte des Grenzen Ziehens, d. h. des (spatial) bordering. ${ }^{20}$ Dieser Begriff trägt dem Problem insofern Rechnung als er „Praktiken des Ordnens und der diskursiven Differenzierungen zwischen uns und den anderen“ ${ }^{21}$ als Praktiken versteht, die sich räumlicher Mittel bedienen, um sozialweltliche Differenzierungen manifest und sichtbar zu machen. ${ }^{22}$

Im disziplinären Kontext der Geographie spiegelt sich in dieser begrifflichen Differenzierung auch die Suche nach einem breiter angelegten, sozialtheoretisch kompatiblen Grenzbegriff wider, der das „klassische geographische Untersuchungsobjekt der Grenze“23 in eine disziplinübergreifende Debatte über die Funktion und Bedeutung des Treffens und Markierens von Unterscheidungen und Differenzen einordnet. Die räumlich markierte und auf diese Weise in die Landschaft eingeschriebene Grenze ist demnach eine spezifische - nämlich mit räumlichen Mitteln vollzogene und oft als selbstverständlich und gegeben erscheinende - Manifestation von gesellschaftlich durchgesetzten Unterscheidungen.

Die Macht staatlicher Grenzen resultiert aus der permanenten wechselseitigen Bestätigung territorialer Markierung und sozialweltlicher Ordnungsfunktion, durch die die kategorialen Ordnungen der sozialen Welt räumlich festgeschrieben

18 Vgl. für den Rio Grande S. S. Mumme, „Advancing Binational Cooperation in Transboundary Aquifer Management on the US-Mexico Border“, Colorado Journal of International Environmental Law and Policy 16 (2005) 77. Die aus der Überlagerung eines Aquifers mit einer politischen Grenze resultierende Komplexitätssteigerung firmiert in der Literatur bei einigen Autor^innen als hydroschizophrenia; vgl. T. Jarvis u. a., „International Borders, Ground Water Flow, and Hydroschizophrenia“, Groundwater 43 (2005) 5, S. 764-770.

19 So z. B. M. T. Klare, „The New Geography of Conflict“, Foreign Affairs (2001), S. 49-61.

20 H. van Houtum, „, The Geopolitics of Borders and Boundaries“, Geopolitics 10 (2005) 4, S. 672 679.

21 Im Original: „practices of ordering and the discursive differentiation between us and them“.

22 Darin unterscheidet sich diese Herangehensweise fundamental von typologisierenden Ansätzen, die Grenzen und Grenzräume anhand der ihnen zugeschriebenen Eigenschaften klassifizieren (so beispielsweise bei O. J. Martinez, Border People: Life and Society in the US-Mexico Borderland, Tucson: University of Arizona Press, 1994). Zu Recht weist Von Bredow darauf hin, dass mancher dieser typologisierenden Ansätze, wie z. B. von Haushofer 1929, aus heutiger Zeit mehr als befremdlich erscheinen (W. Von Bredow, Grenzen. Eine Geschichte des Zusammenlebens vom Limes bis Schengen, Darmstadt: Theiss, 2014, S. 21f.).

23 M. Redepenning, Wozu Raum? Systemtheorie, critical geopolitics und raumbezogene Semantiken, Leipzig: Leibniz-Institut für Länderkunde, 2006, hier S. 26. 
und bestätigt werden. ${ }^{24}$ Grenzen haben mithin eine über ihre unmittelbare Funktion Mobilitäten zu kontrollieren hinausgehende, strukturierende Wirkung im Hinblick auf die grundlegende, ideologisch leicht $\mathrm{zu}$ mobilisierende Dichotomie des Wir hier/Andere dort. ${ }^{25}$ Erst indem der analytische Fokus von der border zum bordering verschoben wird, kann die Funktionsweise der Reifizierungsmaschine „Grenze“ offen gelegt werden.

24 F. Meyer, „Scale up, Harry! Discursive Transition (and Continuity) in the EU's 'Area of Freedom, Security and Justice’ Between 1995 and 2014“, in: B. Bruns, D. Happ, H. Zichner (Hrsg.), European Neighbourhood Policy. Geopolitics Between Integration and Security, London: Palgrave Macmillan, 2016, S. 23-45; C. Johnson, „Competing Para-Sovereignties in the Borderlands of Europe“, Geopolitics 22 (2017) 4, S. 772-793.

25 M. Redepenning, Wozu Raum?. 